\title{
Short-term safety and efficacy of transcarotid transcatheter aortic valve implantation with balloon-expandable vs. self-expandable valves
}

\author{
Damian Hudziak ${ }^{1}$, Wojciech Wańha², Radosław Gocoł ${ }^{1}$, Radosław Parma ${ }^{2}$, Andrzej Ochała ${ }^{2}$, \\ Grzegorz Smolka², Joanna Ciosek ${ }^{2}$, Tomasz Darocha ${ }^{3}$, Marek A. Deja' ${ }^{1}$, Wojciech Wojakowski \\ ${ }^{1}$ Department of Cardiac Surgery, Medical University of Silesia, Katowice, Poland \\ ${ }^{2}$ Division of Cardiology and Structural Heart Diseases, Medical University of Silesia, Katowice, Poland \\ ${ }^{3}$ Department of Anaesthesiology and Intensive Care, Medical University of Silesia, Katowice, Poland
}

Adv Interv Cardiol 2021; 17, 1 (63): 75-81 DOI: https://doi.org/10.5114/aic.2021.104772

\begin{abstract}
A bstract
Introduction: Transfemoral access (TF) is the preferred access for transcatheter aortic valve implantation (TAVI). Transcarotid TAVI (TC-TAVI) is an alternative for patients in whom TF-TAVI is impossible. Two types of valves - balloon-expandable (BE) and self-expandable (SE) - can be used in TC-TAVI procedures.

Aim: Comparison of the short-term results of patients treated with TC-TAVI using BE and SE valves.

Material and methods: The retrospective registry included 39 patients in whom the TC-TAVI procedure was performed between 2017 and 2020 (BE-TAVI; $n=10$, SE-TAVI; $n=29$ ). Preoperative characteristics, operative and postoperative results, and 30-days mortality were compared.

Results: Patients from the BE-TAVI group had higher surgical risk (EuroSCORE) (10.8\% (6.2-14.0) vs. 5.5\% (4.3-8.7); $p=0.027)$. The incidence of chronic obstructive pulmonary disease (COPD) was higher in the SE-TAVI group ( $34.5 \% \mathrm{vs.} 0 \% ; p=0.040)$. In terms of other comorbidities, demographics, preprocedural laboratory results, transthoracic echocardiography (TTE), and multislice computed tomography (MSCT), the two groups were comparable. In both groups, we observed 100\% procedural success. The median valve size was larger in the SE-TAVI group (29.0 (26.0-29.0) vs. 26.0 (23.0-26.0); $p<0.001)$. The hospitalization time was shorter in the BE-TAVI group vs. SE-TAVI $(5.8 \pm 0.6$ vs. $6.4 \pm 0.9 ; p=0.043)$. We did not observe statistically significant differences between BE-TAVI and SE-TAVI in periprocedural and 30-day mortality, or the number of strokes/TIA. Also TTE parameters and NYHA class showed similar improvement at 30 days in both groups.
\end{abstract}

Conclusions: TC-TAVI using balloon-expandable and self-expandable valves showed similar safety and efficacy in 30 days follow-up.

Key words: aortic stenosis, transcarotid access, transcatheter aortic valve implantation, balloon-expandable valve, self-expandable valve.

S u m m a ry

Both self-expanding and balloon-expandable prostheses are used in transcatheter aortic valve implantation (TAVI). Many studies assessing the early safety of TAVI procedures depending on the type of valve prostheses (balloon-expandable and self-expandable) concern femoral access. To our knowledge, there are no studies evaluating the impact of the type of prosthesis on the safety and efficacy of transcarotid TAVI. However, it is known that the access through the common carotid artery may be an alternative for patients in whom femoral access is not available.

\section{Introduction}

Transcatheter aortic valve implantation (TAVI) has become a standard of care for patients with severe symp- tomatic aortic stenosis (AS) who are not eligible for conventional surgical aortic valve replacement (SAVR) and have high surgical risk. Also, based on the heart team decision it is an accepted treatment for intermediate

Corresponding author:

Damian Hudziak MD, PhD, Department of Cardiac Surgery, Medical University of Silesia, Katowice, Poland, e-mail: damhud@gmail.com Received: 14.12.2020, accepted: 13.02.2021. 
and low-risk patients with additional factors favoring the non-surgical approach [1-3]. The transfemoral (TF) access is preferred in all patients because it has the lowest complication rate $[4,5]$. However, in $15-20 \%$ of TAVI patients TF is impossible due to concomitant peripheral arterial diseases (PAD) and abnormalities of the thoracic and abdominal aorta, increasing the risk of bleeding and vascular complications [6-8]. Since the first TAVI via the left common carotid artery (CCA) described by Modine et al. in 2010 [9] transcarotid (TC) access has been used as an alternative for patients disqualified from TF-TAVI $[10,11]$. Both balloon-expandable (BE) and self-expandable (SE) valves can be implanted through the CCA; however, no direct comparisons have been published [10-12].

\section{Aim}

The purpose of our registry including all consecutive patients treated with TC-TAVI using BE and SE valves in a large academic institution was to evaluate early safety and efficacy of the procedure depending on the type of implanted valve.

\section{Material and methods}

The study group consisted of patients with severe symptomatic aortic stenosis who underwent the TCTAVI procedure in our hospital between 2017 and 2020. Since we aimed to investigate outcomes in the TCTAVI procedure, patients with only balloon aortic valvuloplasty were excluded from the final analysis. All patients signed written informed consent to the pro-

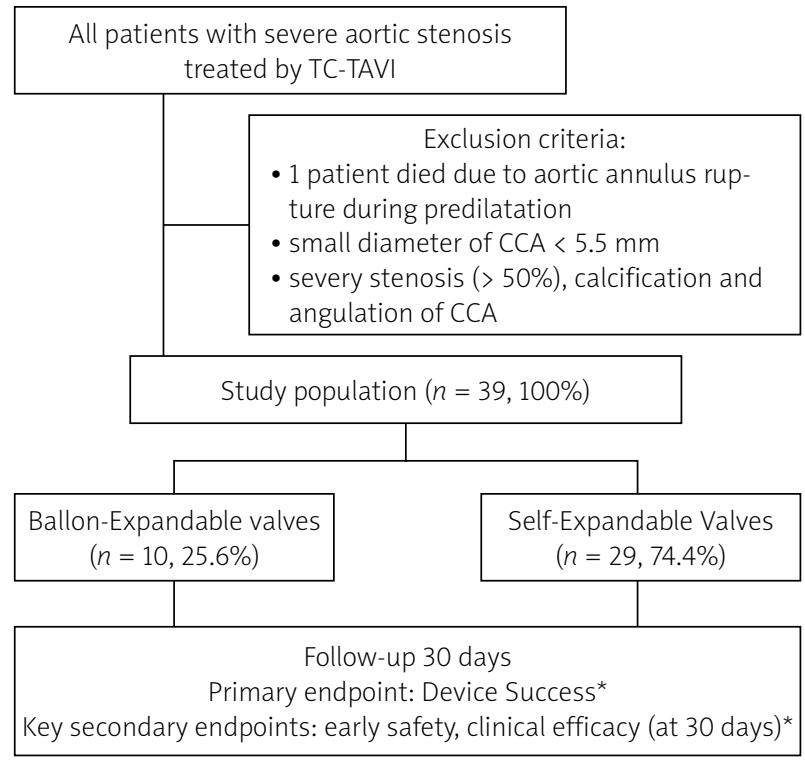

Figure 1 . Schedule of the study groups

TC-TAVI - transcarotid transcatheter aortic valve implantation, CCA - common carotid artery. Balloon-explandable valve: EdwardsSapien 3 Ultra (Edwards Lifesciences Corp. Irvine, CA, USA), Self-explandable valve: Evolute R (Medtronic, Minneapolis, MN, USA) and Portico (Abbott Vascular, Santa Clara, CA, USA). *According to the Valve Academic Research Consortium-2 (VARC-2) consensus. posed treatment. Because of the retrospective study design, no institutional review board permission was required. The results were defined and presented according to the Valve Academic Research Consortium-2 (VARC-2) consensus [13]. The decision on qualification for the TC-TAVI procedure and selection of the size and type of implanted valve prosthesis, BE or SE, was made by the multidisciplinary Heart Team. The access and sizing were planned based on the multislice computed tomography (MSCT) results of the peripheral arteries and aorta, coronary angiography, and transthoracic echocardiography (TTE). MSCT analysis was performed using the 3Mensio (Pie Medical Imaging, The Netherlands) software. The presence of thoracic and abdominal aorta disease (aneurysm, thrombus, chronic dissection, history of a stent graft, tortuosity) as well as peripheral artery disease (PAD), small diameter $(<6 \mathrm{~mm})$, severe calcifications and severe angulation of the iliofemoral arteries disqualified the patient from the femoral access. The MSCT analysis method for carotid access and the operating technique has been described in detail in the previous article [14]. Patients who had CCA diameter more than $5.5 \mathrm{~mm}$ and no significant stenosis (> 50\%), excessive angulation, and calcifications were qualified for TC-TAVI. All procedures were performed in the hybrid operating room during general or local anesthesia by the multidisciplinary TAVI team. Every patient received a pre-procedural intravenous dose of antibiotic (1.5 g cephazolin) to prevent infective endocarditis. Heparin was given in a dose of $100 \mathrm{U} / \mathrm{kg}$ (activated clotting time $>250 \mathrm{~s}$ ). After placement of two 5-0 monofilament continuous purse-string sutures, the 6-Fr vascular sheath was inserted into the carotid artery. The pigtail catheter was positioned in the noncoronary sinus using a 6-Fr sheath inserted through the radial or femoral artery. An endocavitary electrode for rapid ventricular pacing was introduced into the right ventricle through the femoral or jugular vein. Intraoperative monitoring of each patient included regional cerebral oximetry (Covidien, Medtronic plc, Ireland), arterial blood pressure, central venous pressure, saturation of arterial blood, electrocardiography, and transthoracic (TTE) or transoesophageal echocardiography (TOE). Selective carotid angiography was performed to assess arterial patency after the removal of the delivery system.

\section{Statistical analysis}

Categorical data were presented as numbers (\%). The Kolmogorov-Smirnov test was used to assess the data distribution. Normally distributed values were presented as mean with standard deviation. Non-normally distributed values were presented as median with $25^{\text {th }}$ and $75^{\text {th }}$ percentile (interquartile range (IQR)). Continuous data were compared by Student's $t$-test or by the Mann-Whitney $U$ test, depending on the distribution. 
Categorical data were analyzed with the $\chi^{2}$ or Fisher's exact test. $P$-values of $<0.05$ were considered statistically significant. The statistical analysis was performed using MedCalc 17.9.2 (MedCalc software).

\section{Results}

Forty patients were qualified for the TC-TAVI procedure from September 2017 to October 2020 in the Upper Silesian Medical Center of the Medical University of Silesia in Katowice, Poland. One patient was exclud- ed from the analysis because he died intraoperatively prior to aortic valve implantation. The cause of death was a rupture of the aortic annulus during predilation. The retrospective study finally enrolled 39 patients (10 (25.6\%) BE-TAVI and 29 (74.4\%) SE-TAVI) (Figure 1). The baseline characteristics of the patients are included in Table I. The groups did not differ in age (74.5 (69-81) vs. 77 (72-84.2); $p=0.349$ ) or gender ( $80 \%$ males vs. $44.8 \%$; $p=0.073)$. All patients were symptomatic with baseline NYHA class $\geq$ III. Patients in SE-TAVI had a higher inci-

Table I. Baseline clinical characteristics of patients

\begin{tabular}{|c|c|c|c|}
\hline Parameter & $\begin{array}{c}\text { BE TAVI } \\
n=10,25.6 \%\end{array}$ & $\begin{array}{c}\text { SE TAVI } \\
n=29,74.4 \%\end{array}$ & $P$-value \\
\hline Age & $74.5(69.0-81.0)$ & $77.0(72.0-84.2)$ & 0.349 \\
\hline Male & $8(80.0)$ & $13(44.8)$ & 0.073 \\
\hline $\mathrm{BMI}\left[\mathrm{kg} / \mathrm{m}^{2}\right]$ & $33(28.0-34.9)$ & $27(23.7-30.2)$ & 0.053 \\
\hline EuroSCORE (\%) & $10.8(6.2-14.0)$ & $5.5(4.3-8.7)$ & 0.027 \\
\hline $\mathrm{NYHA} \geq \mathrm{III}$ & $10(100)$ & $29(100)$ & - \\
\hline Hypertension & $10(100)$ & $29(100)$ & - \\
\hline Dyslipidemia & $6(60.0)$ & $24(82.8)$ & 0.197 \\
\hline Diabetes & $4(40)$ & $15(51.7)$ & 0.716 \\
\hline COPD & $0(0)$ & $10(34.5)$ & 0.040 \\
\hline PAD & $6(60)$ & $11(37.9)$ & 0.281 \\
\hline Prior MI & $4(40)$ & $9(31.0)$ & 0.704 \\
\hline Prior $\mathrm{PCl}$ & $3(30.0)$ & $13(44.8)$ & 0.479 \\
\hline Prior cardiac surgery & $2(20)$ & $9(31)$ & 0.692 \\
\hline Creatinine level & $1.2(1.0-1.2)$ & $1.0(0.8-1.2)$ & 0.187 \\
\hline GFR $\left[\mathrm{ml} / \mathrm{min} / 1.73 \mathrm{~m}^{2}\right]$ & $59.5(59.0-71.0)$ & $61.0(46.2-71.0)$ & 0.809 \\
\hline Hemoglobin level [g/dl] & $13.1(12.5-14.1)$ & $12.9(11.4-14.2)$ & 0.617 \\
\hline Hematocrit (\%) & $39.7(38.9-41.2)$ & $40.6(34.4-42.4)$ & 0.784 \\
\hline WBC level $\left[\times 10^{3} / \mu \mathrm{l}\right]$ & $6.7(5.2-8.5)$ & $7.1(6.4-8.5)$ & 0.584 \\
\hline PLT level $\left[\times 10^{3} / \mu \mathrm{l}\right]$ & 173.5 (145.0-218.0) & $183.0(142.5-232.2)$ & 0.747 \\
\hline TNT level [ng/ml] & $0.03(0.01-0.04)$ & $0.01(0.01-0.02)$ & 0.025 \\
\hline \multicolumn{4}{|l|}{ ECHO parameters: } \\
\hline$M R>$ moderate & $5(50.0)$ & 9 (31.0) & 0.445 \\
\hline AVA $\left[\mathrm{cm}^{2}\right]$ & $0.6(0.56-0.70)$ & $0.7(0.6-0.8)$ & 0.157 \\
\hline LVEF [\%] & $55.0(40.0-60.0)$ & $50.0(45.0-55.0)$ & 0.625 \\
\hline $\mathrm{PG} \max [\mathrm{mm} \mathrm{Hg}]$ & $83.0(67.0-91.0)$ & $66.0(57.2-81.5)$ & 0.100 \\
\hline $\mathrm{PG}$ mean $[\mathrm{mm} \mathrm{Hg}]$ & $45.0(41.0-57.0)$ & $39.0(35.0-47.2)$ & 0.166 \\
\hline $\operatorname{Vmax}[\mathrm{m} / \mathrm{s}]$ & $4.3(4.0-4.8)$ & $4.0(3.7-4.4)$ & 0.186 \\
\hline \multicolumn{4}{|l|}{ MSCT parameters: } \\
\hline LCCA diameter [mm] & $6.6(5.8-7.8)$ & $6.3(6.0-7.2)$ & 0.910 \\
\hline RCCA diameter [mm] & $6.9(6.0-8.0)$ & $7.2(6.2-8.1)$ & 0.459 \\
\hline BAV & $3(30)$ & $8(27.6)$ & 1.0 \\
\hline LM height [mm] & $14.9(13.1-17.0)$ & $12.9(11.6-14.7)$ & 0.071 \\
\hline RCA height [mm] & $19.4(17.8-20.6)$ & $17.7(15.2-19.6)$ & 0.143 \\
\hline Annulus perimeter [mm] & $79.2(72.9-80.4)$ & $76.8(70.8-80.7)$ & 0.541 \\
\hline $\mathrm{AVAA}\left[\mathrm{mm}^{2}\right]$ & $490(410-499)$ & $460(389-522)$ & 0.846 \\
\hline
\end{tabular}

Data are given as the median (interquartile range) or as $n$ (\%). AVA - aortic valve area, AVAA - aortic valve annulus area, BAV-bicuspid aortic valve, BE - balloon-expandable, BMI - body mass index, COPD - chronic obstructive pulmonary disease, GFR - glomerular filtration rate, LCCA - left common carotid artery, LM - left main coronary artery, LVEF - left ventricular ejection fraction, MI - myocardial infarction, MR - mitral regurgitation, MSCT-multislice computed tomography, NYHA - New York Heart Association, $P A D$ - peripheral arterial disease, $P C I$ - percutaneous coronary intervention, $P G$ max - aortic valve maximal gradient, $P G$ mean - aortic valve mean gradient, $P L T$ - platelets, $R C A$ - right coronary artery, $R C C A$ - right common carotid artery, SE - self-expandable, TAVI - transcatheter aortic valve implantation, TNT - troponin T, Vmax - transaortic peak instantaneous velocity, WBC - white blood cells. 
dence of chronic obstructive pulmonary disease (COPD) (34.5\% vs. $0 \% ; p=0.040)$. The groups did not differ significantly in terms of other comorbidities although the calculated risk of mortality according to the EuroSCORE was higher in the BE-TAVI group (10.8\% (6.2-14.0) vs. $5.5 \%(4.3-8.7) ; p=0.027)$. The preoperative blood test results (level of creatinine, glomerular filtration rate (GFR), hemoglobin, hematocrit, white blood cells (WBC), platelets (PLT)) were comparable. Also, echocardiographic parameters (left ventricular ejection fraction (LVEF); aortic valve maximal gradient (PG max); aortic valve mean gradient (PG mean); aortic valve area (AVA); transaortic peak instantaneous velocity (Vmax); mitral regurgitation $(M R)>2$ grade) and MSCT parameters (diameter left and right CCA, the occurrence of bicuspid aortic valves (BAV); height of left main coronary artery (LM) and right coronary artery (RCA); perimetry; aortic valve annulus area (AVAA)) were comparable between groups. The following valve types were used: Edwards-Sapien 3 Ultra (Edwards Lifesciences Corp. Irvine, CA, USA) in the BE-TAVI group; Evolut R (Medtronic, Minneapolis, MN, USA) and Portico (Abbott Vascular, Santa Clara, CA, USA) in the SE-TAVI group. The prostheses' characteristics are presented in Table II. The size of the implanted BE valves was smaller than the SE valves (26.0 (23.0-26.0) vs. 29.0 (26.0-29.0); $p<0.001)$. All balloon-expandable valves were implanted through a dedicated vascular sheath, while in the self-expandable valves group, the vascular sheath was used only in 7 patients. Perioperative and postoperative results are shown in Table III. 100\% device success was observed in both groups. There were no procedural deaths in patients in whom the valve was implanted. All of BE-TAVI and 93.1\% of SE-TAVI were performed under general anesthesia $(p=1.000)$. Coronary occlusion, prosthetic dislocations and dysfunction which required conversion to SAVR were not observed in either group. There was a similar number of other events defined by VARC-2: myocardial infarction, major vascular complication, life-threatening

Table II. Valves' characteristics

\begin{tabular}{|c|c|c|}
\hline Variable & Prosthesis size $(n)$ & Sheath size $(n)$ \\
\hline \multicolumn{3}{|c|}{ SE TAVI $n=29,74.4 \%$ : } \\
\hline Evolute $\mathrm{R}$ & $26(10)$ & $14(2)$ \\
\hline Evolute R & $29(13)$ & $14(6)$ \\
\hline Evolute R & $34(5)$ & $16(1)$ \\
\hline Portico & $27(1)$ & $16(0)$ \\
\hline \multicolumn{3}{|c|}{ BE TAVI $n=10,25.6 \%$} \\
\hline Sapien 3 Ultra & $23(3)$ & $14(3)$ \\
\hline Sapien 3 Ultra & $26(6)$ & $14(6)$ \\
\hline Sapien 3 Ultra & $29(1)$ & $16(1)$ \\
\hline
\end{tabular}

BE-balloon-expandable, SE-self-expandable, TAVI-transcatheter aortic valve implantation. The size of the vascular sheath on a French scale. Edwards-Sapien 3 Ultra (Edwards Lifesciences Corp., Irvine, CA, USA), Evolute R (Medtronic, Minneapolis, MN, USA), Portico (Abbott Vascular, Santa Clara, CA, USA). bleeding, and acute renal failure in BE and SE groups (Table III). Most of the procedures were performed through the LCCA and a minority through the RCCA (9/10 BE and $28 / 29$ SE). The procedural time, as well as the percentage of balloon aortic pre- and postdilatation (10\% vs. $6.9 \%$; $p=1.000$ and $10 \%$ vs. $20.7 \% ; p=0.652$ respectively), was not significantly different between the groups. A low rate of neurological complications was observed in both the BE-TAVI and SE-TAVI group (no strokes and only 1 TIA (10\%) vs. 2 (6.9\%); $p=0.753$, respectively) (Table III). These data confirm cerebral oximetry results bilaterally during the procedure (Table IV, Figure 1). The time patients spent in the ICU was similar, while the total hospitalization time was shorter in the BE-TAVI group ( $5.8 \pm 0.6$ days vs. $6.4 \pm 0.9$ days; $p=0.043$ ). There was no statistically significant difference in the percentage of atrial fibrillation ( $0 \%$ vs. $6.9 \% ; p=1.000$ ) or pacemaker implantation (10\% vs. $13.8 \%$; $p=1.000$ ). Comparison of the postoperative echocardiography parameters (EF, PG max, PG mean) and the NYHA functional class showed no significant differences between the groups. (Table II). Regardless of the types of prostheses, we noted a similar reduction of AV gradient (PG max and PG mean) from 68.0 (60.2-87.5) to 15.0 (13.0-20.0); $p<0.001$ and from 41.0 (35.2-50.2) to $8.0(6.2-10.0) ; p<0.001$ respectively, with no effect on EF (50.0 (45.0-55.0) vs. 55.0 (50.0-60.0); $p=0.318)$. Also there was an improvement of NYHA class at follow-up (3 $(3-3)$ vs. $2(1-2) ; p<0.001)$. The type of the valve prosthesis did not affect 30-day mortality (0 (0\%) vs. $2(6.9 \%) ; p=1.000$, respectively) in the $\mathrm{BE}$ and SE group. One patient died of an unknown cause 2 weeks after discharge, while the second died 3 weeks after discharge from the hospital due to complications after COVID-19 infection.

\section{Discussion}

TAVI through the femoral artery is the preferred access most often used due to the safety [15]. Despite the reduction of the size of access sheaths and the modification of the vascular closure devices (Proglide, Manta), not all patients can undergo TF-TAVI and need alternative access sites. In many centers, TC access has become the first choice for patients for whom the femoral approach is not available. In our hospital, the TC-TAVI percentage is approximately $10 \%$ of all TAVI procedures, and it is similar to the result reported by other authors $[16,17]$. The common carotid artery is easy to access because of its superficial location and size. The carotid artery preparation technique is not complicated but requires special attention to protect the jugular vein, the vagus, and the laryngeal nerves. The most common local access complications are injury to the vagus nerve, local hematoma, and wound infection [18]. In our group, of these complications, we observed only local hematoma in 1 patient in the BE group. Cerebrovascular events re- 
Table III. Periprocedural and postprocedural outcomes

\begin{tabular}{|c|c|c|c|}
\hline Variable & $\begin{array}{c}\text { BE TAVI } \\
n=10,25.6 \%\end{array}$ & $\begin{array}{c}\text { SE TAVI } \\
n=29,74.4 \% \\
\end{array}$ & $P$-value \\
\hline \multicolumn{4}{|l|}{ Periprocedural: } \\
\hline Device success & $10(100)$ & $29(100)$ & - \\
\hline Procedural mortality & $0(0)$ & $0(0)$ & - \\
\hline Coronary occlusion & $0(0)$ & $0(0)$ & - \\
\hline Prosthesis dislocation & $0(0)$ & $0(0)$ & - \\
\hline Procedural time, min & $70.0(60.0-75.0)$ & $65.0(60.0-80.0)$ & 0.577 \\
\hline General anaesthesia & $10(100)$ & $27(93.1)$ & 1.000 \\
\hline Implantation by LCCA & $9(90)$ & $28(96.6)$ & 0.452 \\
\hline Balloon aortic predilatation & $1(10.0)$ & $2(6.9)$ & 1.00 \\
\hline Balloon aortic postdilatation & $1(10.0)$ & $6(20.7)$ & 0.652 \\
\hline Conversion to SAVR & $0(0)$ & $0(0)$ & - \\
\hline \multicolumn{4}{|l|}{ Postprocedural: } \\
\hline Stroke & $0(0)$ & $0(0)$ & - \\
\hline TIA & $1(10)$ & $2(6.9)$ & 0.753 \\
\hline ICU stay [days] & $2.5(2.0-4.0)$ & $2.0(2.0-3.0)$ & 0.336 \\
\hline Hospital stay [days] & $5.8 \pm 0.6$ & $6.4 \pm 0.9$ & 0.043 \\
\hline Mechanical ventilation time [min] & $120(80.0-180.0)$ & $360(90.0-420.0)$ & 0.213 \\
\hline New pacemaker implantation & $1(10)$ & $4(13.8)$ & 1.0 \\
\hline New atrial fibrillation & $0(0)$ & $2(6.9)$ & 1.0 \\
\hline Myocardial infarction & $0(0)$ & $1(3.4)$ & 1.0 \\
\hline Inotropic drugs & $2(20)$ & $4(13.8)$ & 0.636 \\
\hline Blood transfusion & $2(20)$ & $3(10.3)$ & 0.587 \\
\hline Tamponade & $0(0)$ & $1(3.4)$ & 1.0 \\
\hline Major vascular complication & $1(10)$ & $0(0)$ & 0.256 \\
\hline Life-threatening bleeding & $1(10)$ & $0(0)$ & 0.256 \\
\hline Creatinine level [mg/dl] & $1.0(0.8-1.3)$ & $1.0(0.8-1.3)$ & 0.765 \\
\hline GFR $\left[\mathrm{ml} / \mathrm{min} / 1.73 \mathrm{~m}^{2}\right]$ & $71.0(52.0-79.0)$ & $59.0(46.5-77.7)$ & 0.606 \\
\hline Hemoglobin level [g/dl] & $11.2(10.7-12.0)$ & $11.2(9.8-11.7)$ & 0.498 \\
\hline Hematocrit (\%) & $33.6(31.6-35.2)$ & $33.3(29.4-35.4)$ & 0.630 \\
\hline 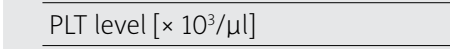 & $129.0(105.0-145.0)$ & $137.0(109.0-162.0)$ & 0.551 \\
\hline TNT level [ng/ml] & $0.16(0.122-0.189)$ & $0.13(0.106-0.209)$ & 0.421 \\
\hline WBC level $\left[\times 10^{3} / \mu \mathrm{l}\right]$ & $5.8(4.9-9.5)$ & $6.3(4.5-8.1)$ & 0.834 \\
\hline $\mathrm{PG} \max [\mathrm{mm} \mathrm{Hg}]$ & $16.0(13.0-22.0)$ & $15.0(12.9-19.2)$ & 0.508 \\
\hline $\mathrm{PG}$ mean [mm Hg] & $9(7.0-14.0)$ & $8.0(6.0-10.0)$ & 0.316 \\
\hline $\operatorname{Vmax}[\mathrm{m} / \mathrm{s}]$ & $2.0(1.6-2.3)$ & $2.0(1.8-2.1)$ & 0.948 \\
\hline$P V L$ grade $>2$ & $0(0)$ & $2(6.9)$ & 1.0 \\
\hline LVEF (\%) & $55.0(50.0-60.0)$ & $55.0(48.7-60.0)$ & 0.768 \\
\hline 30-day mortality & $0(0)$ & $2(6.9)$ & 1.0 \\
\hline
\end{tabular}

Table IV. Results of cerebral oximetry during procedure

\begin{tabular}{|c|c|c|c|c|c|c|}
\hline \multirow[t]{2}{*}{ Variable } & \multicolumn{3}{|c|}{ Cerebral oximetry on the implantation side } & \multicolumn{3}{|c|}{ Cerebral oximetry on the opposite side } \\
\hline & $\begin{array}{c}\text { BE TAVI } \\
n=10,25.6 \%\end{array}$ & $\begin{array}{c}\text { SE TAVI } \\
n=29,74.4 \%\end{array}$ & $P$-value & $\begin{array}{c}\text { BE TAVI } \\
n=10,25.6 \%\end{array}$ & $\begin{array}{c}\text { SE TAVI } \\
n=29,74.4 \%\end{array}$ & $P$-value \\
\hline Baseline & $65.0(63.0-68.0)$ & $68.0(62.0-72.0)$ & 0.279 & $65.5(63.0-71.0)$ & $67.0(63.7-74.2)$ & 0.366 \\
\hline Cannulation & $59.0(54.0-63.0)$ & $61.0(55.0-65.0)$ & 0.651 & $64.0(57.0-71.0)$ & $60.0(55.7-71.0)$ & 0.746 \\
\hline Implantation & $39.0(29.0-47.0)$ & $45.0(37.2-47.0)$ & 0.365 & $51.0(31.0-58.0)$ & $49.0(42.7-55.0)$ & 1.000 \\
\hline Post procedure & $68.0(68.0-71.0)$ & $70.0(62.7-75.0)$ & 0.935 & $71.0(70.0-75.0)$ & $70.0(63.0-75.0)$ & 0.519 \\
\hline
\end{tabular}

Data are given as the median (interquartile range). BE - balloon-expandable, SE-self-expandable, TAVI-transcatheter aortic valve implantation. 
lated to the use of the CCA approach, including stroke and TIA, are estimated at $2-4 \%[11,19,20]$. In our registry, there was no stroke, and we observed 3 cases of TIA (7.7\% in 39 patients), which were not related to the type of prosthesis and resolved within $4 \mathrm{~h}$. We believe that the two aspects are very important in preventing severe neurological complications. The first is a detailed analysis of the MSCT during qualification, allowing one to choose an artery with a larger diameter, less calcification, and tortuosity, and with a more favorable spatial relationship between the virtual CCA centerline and the plane of the aortic annulus. It has a significant impact on facilitating and shortening the time of the procedure. Confirmation in the MSCT of the borderline dimensions of CCA allows one to consider using a sheathless technique for the planned SE valve. The second aspect is the continuous monitoring of cerebral oximetry throughout the procedure, which allows for a quick response to possible brain perfusion disorders. In a meta-analysis of Wee et al. [19] (3.8\% neurological complication rate in 364 patients) cerebral oximetry was the main tool for periprocedural cerebral monitoring. Our analysis did not reveal any differences between groups in left and right side cerebral oximetry values in the successive steps of the procedure (Table IV). In addition, in the comparison of oximetry results for all TC-TAVI, the baseline values did not differ from the values after implantation (Figure 2). In our center, during the TC-TAVI procedure, we do not perform a 2-3 min clamping of the carotid artery to check for the Willis circle function, as proposed by some operators [10, 12]. In our surgical technique, the carotid artery was carefully dissected, especially protecting the vagus nerve, and manually examined after dissection for the presence of calcifications. Two 5-0 monofilament continuous purse-string sutures were used similarly to the technique used to cannulate the ascending aorta

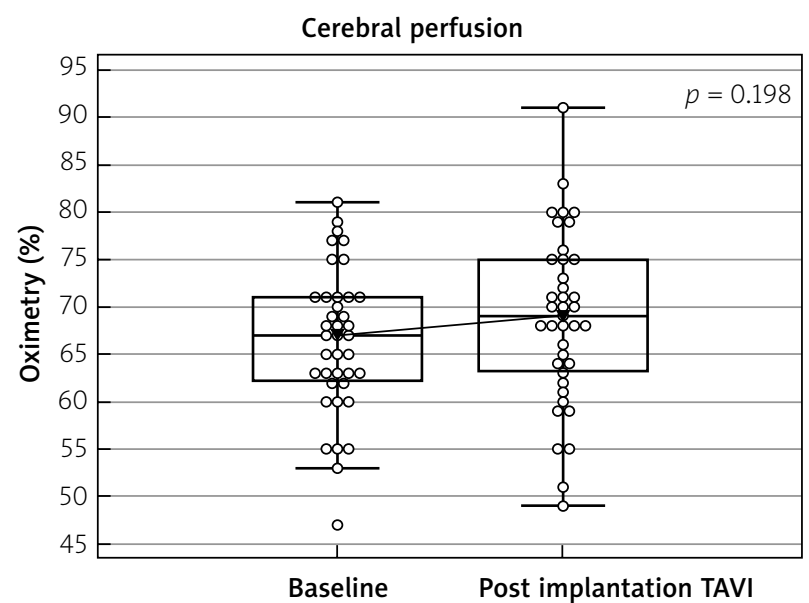

Figure 2. Comparison baseline and post implanation results of the cerebral oximetry all trancarotid TAVI patient $(n=39)$

TAVI - transcatheter aortic valve implantation. during the classical surgical operation. After the procedure, the carotid artery was closed using sutures without clamping. We do not prefer clamping the carotid artery to reduce its trauma. In cerebral oximetry, we did not observe a difference in saturation between the left and right sides after leading the delivery sheath (14, $16 \mathrm{Fr}$ ) (oximetry values were not lower than $55 \%$ ); this allowed the procedure to be continued safely. In our observation, valve selection had no effect on the other endpoints according to the VARC-2 consensus. Percentages of life-threatening bleeding (2.6\%), myocardial infarctions (2.6\%), major vascular complications (2.6\%), acute kidney injuries (0\%), pacemaker implantations (12.8\%), and all mortality $(6.9 \%)$ for all the TC-TAVI group were comparable with the results reported by other authors. Similar results were obtained for the choice of implantation site (LCCA-94.9\% (90\% in the BE group and $96.6 \%$ in the SE group)) and type of anesthesia (general anesthesia $-94.9 \%$ of all TC-TAVI $(100 \%$ in the BE group and $93.1 \%$ in the SE group)) $[11,16,17,19]$. We prefer general anesthesia with intubation because this method increases security by reducing the uncontrolled movement of the patient. However, in special situations, we successfully performed TC-TAVI using SE valves in 2 patients. The results of large analyses comparing the implantation of two types of valves (BE vs. SE) implanted transfemorally are inconclusive. In the French registry comparing the results of the Sapien $3 \mathrm{BE}$ valve with the Evolut R SE valve, 20,918 patients showed lower numbers of all-cause death, cardiovascular death, rehospitalization for heart failure, and pacemaker implantation in the group of patients with a Sapien 3 BE valve [21]. On the other hand, Vlastra et al., analyzing 12381 patients, did not confirm lower 30-day mortality in the BE valve group. They found lower incidence of strokes and pacemaker implantation but more life-threatening bleeding events in these groups [22]. In our analysis, all perioperative and postoperative outcomes in the study groups were comparable. Independent of valve prosthesis type, significant reduction of NYHA class, and improvement of echocardiographic parameters (Pg max, Pg mean, Vmax, and low number of severe ( $>2$ grade) paravalvular leaks $(P V L)$ ) were observed. These data confirmed the good hemodynamic profile of the used prostheses, as well as the adequate size and correct implantation. Paravalvular leak, structural valve deterioration, prosthesis-patient mismatch, leaflet thrombosis, and endocarditis can lead to faster valve dysfunction [23]. Morphometric analysis of aortic valve calcifications in MSCT described by Ryś et al. seems to be an interesting method to reduce the potential risk of PVL in TAVI procedures [24]. The choice between an SE or BE valvular prosthesis depends mainly on the analysis of MSCT (values of the aortic valve annulus area and annulus perimeter, height of the left and right coronary artery, and diameter of the common carotid arteries), type of the native valve (tricuspid vs. bi- 
cuspid), presence of the failed aortic bioprosthetic valve (ViV) and extent of calcifications (especially massive calcifications extending into the left ventricular outflow tract). In addition, in our opinion, in patients who have a smaller size of the common carotid arteries, it is worth using a self-expanding valve because it can be implanted without a dedicated vascular sheath. This may be important for blood flow through the carotid artery and the reduction of neurological complications. In patients with a higher EuroSCORE, we prefer to use BE valve prosthesis implantation because, in our opinion, this system is more predictable, avoids pre- and post-dilatation, and the procedure time is shorter, which may be important for the course and safety of the procedure.

The presented analysis has several limitations. The study was retrospective, non-randomized, and single-center. Only the early results were assessed. Patients in the BE valve group had a higher EuroSCORE II risk. On the other hand, all of the TC procedures were performed consistently according to the institutional standard operating procedures. Importantly, the TC TAVI was always done under brain oxygenation monitoring.

\section{Conclusions}

Based on our registry, we can conclude that transcarotid access is safe and effective, independently of which types of valve prosthesis (balloon-expandable or self-expandable) were used. In our opinion, precise preprocedural multivariate analysis of MSCT and continuous monitoring of cerebral oximetry are key to the success of transcarotid TAVI.

\section{Conflict of interest}

The authors declare no conflict of interest.

\section{References}

1. Cribier A, Eltchaninoff H, Bash A, et al. Percutaneous transcatheter implantation of an aortic valve prosthesis for calcific aortic stenosis. First Human Case Description 2002; 106: 3006-8.

2. Leon M, Mac M, Miller C, et al. Transcatheter aortic-valve implantation for aortic stenosis in patient who cannot undergo surgery. N Engl J Med 2010; 363: 1597-607.

3. Siontis GCM, Praz F, Pilgrim T, et al. Transcatheter aortic valve implantation vs. surgical aortic valve replacement for treatment of severe aortic stenosis: a meta-analysis of randomized trials. Eur Heart J 2016; 37: 3503-12

4. Baumgartner H, Falk V, Bax JJ, et al.; ESC Scientific Document Group. 2017 ESC/EACTS guidelines for the management of valvular heart disease. Eur Heart J 2017; 38: 2739-91.

5. Nishimura RA, Otto CM, Bonow RO, et al. 2017 AHA/ACC Focused Update of the 2014 AHA/ACC Guideline for the Management of Patients With Valvular Heart Disease: A Report of the American College of Cardiology/American Heart Association Task Force on Clinical Practice Guidelines. J Am Coll Cardiol 2017; 70: 252-89.

6. Van Mieghem NM, Tchetche D, Chieffo A, et al. Incidence, predictors, and implications of access site complications with transfemoral transcatheter aortic valve implantation. Am J Cardiol 2012; 110: 1361-7.
7. Grover FL, Vemulapalli S, Carroll J, et al. STS/ACC TVT Registry. 2016 annual report of the Society of Thoracic Surgeons/American College of Cardiology Transcatheter Valve Therapy Registry. J Am Coll Cardiol 2017; 69: 1215-30.

8. Auffret V, Lefevre T, Van Belle E, et al. Temporal trends in transcatheter aortic valve replacement in France: FRANCE 2 to FRANCE TAVI. J Am Coll Cardiol 2017; 70: 42-55.

9. Modine T, Lemesle G, Azzaoui R, et al. Aortic valve implantation with the CoreValve ReValving System via left carotid artery access: first case report. J Thorac Cardiovasc Surg 2010; 140: 928-9.

10. Kirker EB, Hodson RW, Spinelli KJ, et al. The carotid artery as a preferred alternative access route for transcatheter aortic valve replacement. Ann Thorac Surg 2017; 104: 621-9.

11. Mylotte D, Sudre A, Teiger E, et al. Transcarotid transcatheter aortic valve replacement: feasibility and safety. JACC Cardiovasc Interv 2016; 9: 472-80.

12. Debry N, Delhaye C, Azmoun A, et al. Transcarotid transcatheter aortic valve replacement: general or local anesthesia. JACC Cardiovasc Interv 2016; 9: 2113-20.

13. Kappetein AP, Head SJ, Généreux P, et al. Updated standardized endpoint definitions for transcatheter aortic valve implantation: the Valve Academic Research Consortium-2 consensus document. J Thorac Cardiovasc Surg 2013; 145: 6-23.

14. Hudziak D, Nowak A, Gocoł R, et al. Prospective registry on cerebral oximetry-guided transcarotid TAVI in patients with moderate-high risk aortic stenosis. Minerva Cardioangiol 2019; 67: 11-8.

15. Overtchouk P, Modine T. Alternate access for TAVI: stay clear of the chest. Interv Cardiol 2018; 13: 145-50.

16. Watanabe M, Takahashi S, Yamaoka H, et al. Comparison of transcarotid vs. transfemoral transcatheter aortic valve implantation. Circ J 2018; 82: 2518-22.

17. Folliguet TA, Teiger E, Beurtheret S, et al. Carotid versus femoral access for transcatheter aortic valve implantation: a propensity score inverse probability weighting study. Eur J Cardiothorac Surg 2019; 56: 1140-6.

18. North American Symptomatic Carotid Endarterectomy Trial Collaborators; Barnett HJM, Taylor DW, et al. Beneficial effect of carotid endarterectomy in symptomatic patients with high-grade carotid stenosis. N Engl J Med 1991; 325: 445-53.

19. Wee IJY, Stonier T, Harrison M, et al. Transcarotid transcatheter aortic valve implantation: a systematic review. J Cardiol 2018; 71: 525-33.

20. Chamandi C, Abi-Akar R, Rodés-Cabau J, et al. Transcarotid compared with other alternative access routes for transcatheter aortic valve replacement. Circ Cardiovasc Interv 2018; 11: e006388.

21. Deharo P, Bisson A, Herbert J, et al. Impact of Sapien 3 Balloon-Expandable Versus Evolut R self-expandable transcatheter aortic valve implantation in patients with aortic stenosis: data from a nationwide analysis. Circulation 2020; 28: 260-8.

22. Vlastra W, Chandrasekhar J, Muñoz-Garcia AJ, et al. Comparison of balloon-expandable vs. self-expandable valves in patients undergoing transfemoral transcatheter aortic valve implantation: from the CENTER-collaboration. Eur Heart J 2019; 40: 456-65.

23. Piroli F, Franchin L, Bruno F, et al. New advances in the prevention of transcatheter aortic valve implantation failure: current and future perspectives. Kardiol Pol 2020; 78: 842-9.

24. Ryś M, Hryniewiecki T, Michałowska I, et al. Quantitative estimation of aortic valve calcification in multislice computed tomography in predicting the development of paravalvular leaks following transcatheter aortic valve replacement. Adv Interv Cardiol 2018; 14: 85-9. 